\title{
Effects of Sound-Induced Hearing Loss and Hearing Aids on the Perception of Music
}

\author{
BRIAN C. J. MOORE, AES Member \\ (bcjm@cam.ac.uk) \\ Department of Experimental Psychology, University of Cambridge, UK
}

\begin{abstract}
Exposure to high-level music produces several physiological changes in the auditory system that lead to a variety of perceptual effects. Damage to the outer hair cells within the cochlea leads to a loss of sensitivity to weak sounds, loudness recruitment (a more rapid than normal growth of loudness with increasing sound level), and reduced frequency selectivity. Damage to inner hair cells and/or synapses leads to degeneration of neurons in the auditory nerve and to a reduced flow of information to the brain. This leads to poorer auditory discrimination and may contribute to reduced sensitivity to the temporal fine structure of sounds and to poor pitch perception. Hearing aids compensate for the effects of threshold elevation and loudness recruitment via multichannel amplitude compression, but they do not compensate for reduced frequency selectivity or loss of inner hair cells/synapses/neurons. Multichannel compression can impair some aspects of the perception of music, such as the ability to hear out one instrument or voice from a mixture. The limited frequency range and irregular frequency response of most hearing aids is associated with poor sound quality for music. Finally, systems for reducing acoustic feedback can have undesirable side effects when listening to music.
\end{abstract}

\section{INTRODUCTION}

Fig. 1 shows a cross-section of the cochlea, which forms part of the inner ear. Sound evokes a traveling wave on the basilar membrane (BM) and the position of peak vibration varies systematically with the input frequency. The vibration pattern around the peak is amplified and sharpened by an active mechanism that depends on the integrity of the outer hair cells (OHCs); these form 3-5 rows running along the length of the BM. The vibration is detected via the inner hair cells (IHCs), which form a single row running along the length of the BM. Electrical currents flowing through the IHCs lead to a release of neurotransmitter that in turn leads to activity in the neurons that make up the auditory nerve, via the synapses of these neurons on the IHCs.

Exposure to high-level sounds, including music, can lead to permanent damage to or dysfunction of the OHCs, the IHCs, the synapses between the IHCs and neurons, and the neurons [1-3]. The perceptual consequences of each of these different forms of damage are described in the following sections. However, it should be noted that, usually, more than one of these forms of damage is involved $[4,5]$.

\section{PERCEPTUAL CONSEQUENCES OF SOUND-INDUCED DAMAGE}

\subsection{OHC Damage}

Damage to the OHCs impairs the operation of the active mechanism, which has three perceptual consequences.
First, it reduces the amount of BM vibration around the peak of the vibration pattern. The perceptual correlate of this is an elevation in the absolute threshold - the lowest detectable sound level. The maximum amplification produced by the active mechanism is about $55 \mathrm{~dB}$ [6], so the maximum hearing loss that can be produced by $\mathrm{OHC}$ damage alone is also about $55 \mathrm{~dB}$. Hearing loss produced by exposure to intense sounds, including music, is typically greatest over the frequency range 3-6 kHz. As a consequence, weak highfrequency sounds (e.g., high notes played by a piccolo) may not be detected, and low-level musical sounds may have a "muffled" or "dull" timbre, since the higher harmonics may be inaudible.

A second consequence of OHC damage is reduced frequency selectivity. Each point on the BM behaves like a bandpass filter; the center frequency $(\mathrm{CF})$ of the filter varies with position along the BM. These filters, sometimes called the auditory filters, have bandwidths at medium and high CFs that are $12-13 \%$ of the $\mathrm{CF}$ for people with normal hearing [8]. Damage to the OHCs causes these filters to broaden by a factor up to 4 [2]. This reduces the ability of the auditory system to determine the spectral shape of sounds, which is important for distinguishing spoken or sung speech sounds and for distinguishing different musical instruments. It also reduces the ability to "hear out" one sound in the presence of other sounds [9] and increases the susceptibility to masking from background sounds [10].

A third consequence of OHC damage is an effect called loudness recruitment [11]. Assume that the level of a sound 


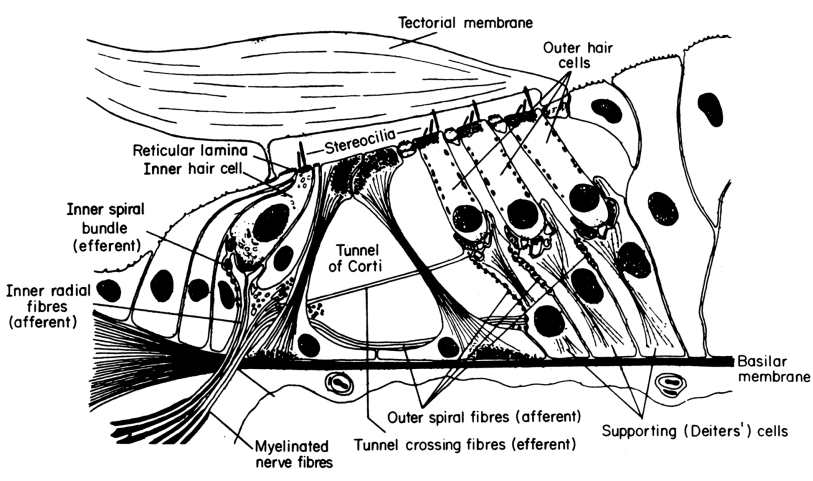

Fig. 1. Cross-section of the cochlea. From [7].

is progressively increased from a low starting value. Once the sound level exceeds the elevated absolute threshold, the loudness grows more rapidly than normal. At high sound levels, the loudness in an ear with $\mathrm{OHC}$ damage "catches up" with the loudness in a normal ear. This is illustrated in Fig. 2, which shows results obtained using a listener with a hearing loss in the right ear and near-normal hearing in the left ear. A pure tone with a frequency of $2.5 \mathrm{kHz}$ was presented in alternation to the two ears, and the listener was asked to adjust the level of the tone in one ear so that its loudness matched that of a fixed-level tone in the other ear.

When the tone in the right ear was presented at $80 \mathrm{~dB}$ SPL, it was matched by a tone in the left ear at about 37 $\mathrm{dB}, \mathrm{a} 43-\mathrm{dB}$ difference. When the level at the right ear was $98 \mathrm{~dB}$ SPL, the matching level in the left ear was about 94 $\mathrm{dB}$ SPL, a difference of only $4 \mathrm{~dB}$. A complementary way of describing loudness recruitment is in terms of dynamic range. For a person with normal hearing, the range between the threshold for detecting a sound and the level at which it becomes unpleasantly loud is typically about $100 \mathrm{~dB}$. For a person with substantial $\mathrm{OHC}$ damage, the range may be only $20-30 \mathrm{~dB}$.

Loudness recruitment has effects similar to multichannel fast-acting amplitude expansion [13, 14]. One perceptual result is that fluctuations in sound level appear exaggerated. For example, an amplitude-modulated sound appears to fluctuate more when presented to an ear with loudness recruitment than when presented to a normal ear [15]. Loudness recruitment tends to be greatest for frequencies where the hearing loss is greatest. Hence, for hearing loss produced by exposure to intense sounds, the effects of recruitment are greatest for sounds with frequencies between 3 and $6 \mathrm{kHz}$. Such sounds may appear to jump abruptly in loudness as the level of the sounds changes [16].

Another possible consequence of damage to the $\mathrm{OHCs}$ is that anomalies in pitch perception can occur. For example, a pure tone of fixed frequency presented in alternation to the two ears may appear to have a different pitch in the left and right ears. This effect is called binaural diplacusis $[17,18]$. It may happen because the position of the peak of the traveling wave on the basilar membrane evoked by a pure tone depends on the operation of the active mechanism and can shift when the function of the OHCs is impaired $[19,20]$. Hearing aids do not compensate for the effects of binaural diplacusis.

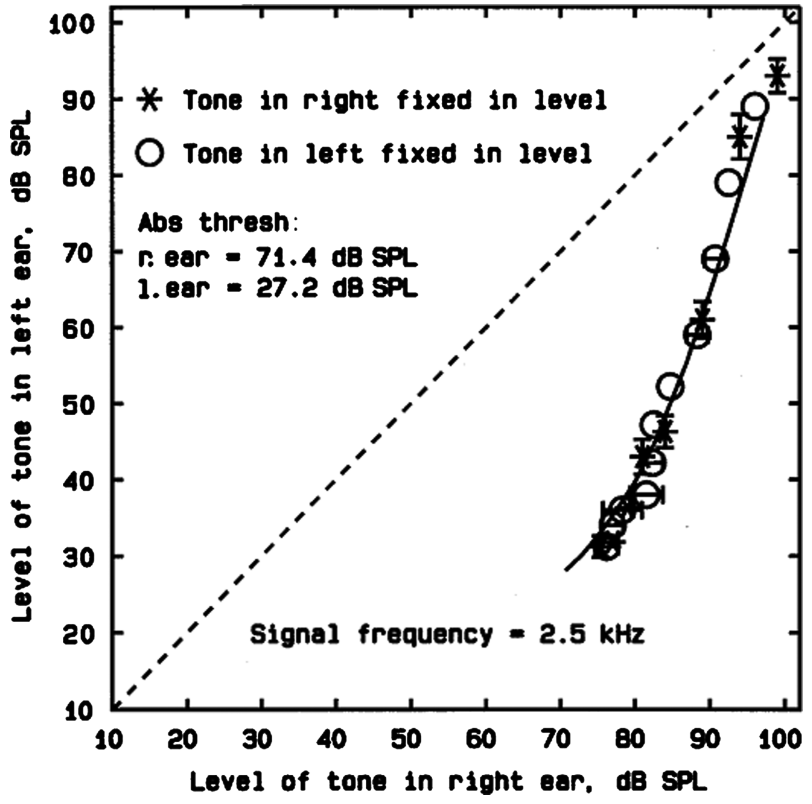

Fig. 2. Results of loudness matching across ears for a listener with unilateral hearing loss. Data from [12].

\subsection{IHC, Synaptic, and Neural Damage}

IHC damage may lead to a disruption of the temporal synchrony between the waveform on the BM and the action potentials (spikes) in the auditory nerve, although this effect appears to be small in animal models of noise-induced hearing loss [21, 22]. IHC, synaptic, and neural damage can all reduce the number of nerve spikes transmitted along the auditory nerve, leading to less precise neural coding of the properties of sounds. This can cause poorer discrimination of sounds and may contribute especially to reduced sensitivity to the temporal fine structure of sounds and to poor pitch perception and sound localization [22].

When the IHC, synaptic, or neural damage is nearly complete over a certain region along the BM, little or no information about BM vibration in that region is conveyed in the auditory nerve. I refer to such a region as a "dead region" $[23,24]$. Dead regions are probably not common among classical musicians, but may be common among rock musicians or those who regularly attend rock concerts or discotheques, who are generally exposed to higher sound levels [25]. Tones falling within a dead region are often perceived as highly distorted or noise-like [26] and often do not have a clear pitch [27].

\subsection{Other Perceptual Effects}

Dysfunction of OHCs, IHCs, and/or neurons leads to a reduced neural input to the central auditory system. It appears that in response to this the central auditory system applies greater gain to the signal coming from the auditory nerve, and this in turn may lead to two perceptual effects: tinnitus, the perception of sound in the absence of any measurable physical sound; and hyperacusis, an increased sensitivity to sounds of medium and high levels [28, 29]. Tinnitus may occur because the increased central gain amplifies various 
forms of neural noise that would not normally be audible. Hyperacusis may arise because the greater gain applied to medium and high-level inputs results in a greater-thannormal loudness. Whatever the causes, both tinnitus and hyperacusis can be troublesome for musicians. Tinnitus may be especially disturbing during piano passages in music, while hyperacusis may make the listening experience unpleasant during forte passages. There is at present no cure for tinnitus, although hearing aids are sometimes prescribed as part of therapy for alleviating the distress caused by tinnitus, and some hearing aids can generate special sounds designed to alleviate tinnitus [30]. Hearing aids are not generally recommended for people with hyperacusis [31], although some hearing aids can be programmed to attenuate high-level sounds, which might in theory provide some relief from hyperacusis.

\section{WHAT CAN HEARING AIDS DO?}

\subsection{Compensation for Threshold Elevation and Loudness Recruitment}

Most current hearing aids compensate at least partly for the effects of threshold elevation and loudness recruitment. Threshold elevation can be compensated using amplification, and loudness recruitment can be compensated using automatic gain control (AGC), also called amplitude compression. Since sound-induced hearing loss leads to the greatest threshold elevation and loudness recruitment over the range 3-6 kHz, more gain and compression are needed over that range than at lower frequencies. This is achieved in hearing aids by splitting the input signal into multiple frequency channels (typically between 2 and 24) and applying independent amplification and amplitude compression in each channel. It should be noted that the number of channels needed to compensate for changes in loudness recruitment with frequency is much less than 24 . However, it is possible use the flexibility provided by many channels to achieve the target frequency response more accurately, and to partially compensate for irregularities in frequency response (see Sec. 5). Also, many channels may be beneficial for other aspects of signal-processing, such as noise reduction or adaptive directionality [32].

Fig. 3 illustrates the basic idea of AGC. For low input levels, the gain is independent of input level; the input-output function has a slope of one. For higher input levels, the gain decreases with increasing input level, and the input-output function has a slope less one. The compression threshold is defined as the input level at which the gain is $2 \mathrm{~dB}$ lower than that applied in the region of linear amplification [33]. For very high input levels, many hearing aids apply infinite compression, also called output limiting, to prevent loudness discomfort. One reason for having a compression threshold is that it is impractical to continue to increase the gain indefinitely as the input level decreases; this would often lead to acoustic feedback for very low input levels (see Sec. 6). A second reason is that the use of high gain for very low-level inputs can make microphone noise or low-level environmental noise appear intrusive. Indeed, for input lev-

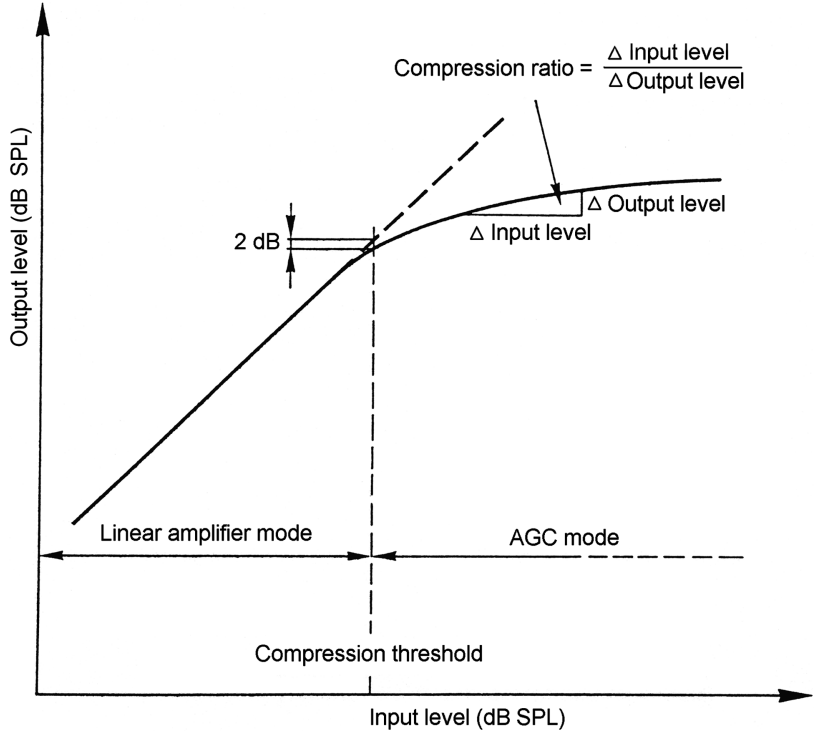

Fig. 3. Typical input-output function for one channel of a hearing aid.

els below about $25-30 \mathrm{~dB}$ SPL, most hearing aids reduce the gain to prevent such noises from being audible; this is called low-level expansion. Hence, hearing aids never fully restore audibility to "normal."

The speed of response of the AGC in a hearing aid is usually measured by using as input a sound whose level changes abruptly between two values, 55 and $90 \mathrm{~dB}$ SPL [33]. When the sound level abruptly increases, the gain decreases, but this takes time to occur. Hence the output of the system shows an initial "overshoot," followed by a decline to a steady value. The time taken for the output to get within $3 \mathrm{~dB}$ of its steady value is called the attack time, $t$ a. When the sound level abruptly decreases, the gain increases, but again this takes time to occur. Hence the output of the system shows an initial dip or "undershoot," followed by an increase to a steady value. The time taken for the output to increase to within $4 \mathrm{~dB}$ of its steady value is called the recovery time or release time, $t$ r.

AGC systems in hearing aids can be divided into two broad classes. The first is intended to adjust the gain automatically for different listening situations. Essentially, such systems relieve the user of the need to adjust the volume control. The gain is changed slowly with changes in input sound level; this is achieved by making the recovery time, or both the recovery time and the attack time, relatively long (usually $t \mathrm{r}$ is between 0.5 and $20 \mathrm{~s}$ ). These systems are often referred to as "automatic volume control" (AVC). The compression ratio in such systems can be high (if the design philosophy is to present all sounds at a comfortable level) or more moderate (if the design philosophy is to give some impression of the overall level of sounds in the environment). AVC systems act almost like linear systems in that they hardly change the gain in response to short-term fluctuations in level for signals such as speech and music.

The second class of AGC system is intended to make the hearing-impaired person's perception of loudness more like that of a normal-hearing listener [34]. Since loudness 
recruitment behaves like fast-acting multichannel expansion, restoration of loudness perception to normal requires fast-acting multichannel compression. Systems with this goal have relatively short attack and recovery times ( $t \mathrm{a}$ is 0.5 to $20 \mathrm{~ms}$ and $t \mathrm{r}$ is $5-200 \mathrm{~ms}$ ). They are often referred to as "fast-acting compressors" or "syllabic compressors," since the gain changes over times comparable to the durations of individual syllables in speech. Fast-acting AGC systems usually have lower compression ratios than AVC systems. High compression ratios (above about 3 ) are avoided, as these have been shown to have deleterious effects on speech intelligibility [35, 36].

Most hearing aids are fitted using a published or manufacturer-specific "prescription method" based on the audiogram of the user [37, 38]. The goal may be to restore loudness perception to "normal" $[39,40]$, to make all mid-frequency bands of speech equal in loudness [41], or to optimize audibility while avoiding uncomfortable loudness [42]. However, the frequency- and level-dependent gains actually achieved can differ markedly from those programmed into the hearing aid. In practice, the gains should be adjusted based on "real-ear measurements" using a small probe microphone placed close to the eardrum [43]. In addition, it is often necessary to make adjustments based on the preferences of the individual in terms of loudness and tone quality.

\subsection{Advantages and Disadvantages of Slow-acting and Fast-acting Compression}

Both slow-acting and fast-acting AGC can be found in hearing aids. Each has advantages and disadvantages. The advantages of slow-acting AGC are:

(1) If desired, signals can be delivered at a comfortable level, regardless of the input level, by use of a high compression ratio.

(2) The temporal envelopes of signals are hardly distorted. This may be important for maintaining speech intelligibility and for hearing individual musical instruments or voices in a mixture [44].

(3) Short-term changes in the spectral patterns of sounds, which convey information in music, are not distorted because the pattern of gains across frequency changes only slowly with time.

(4) Harmonic and inter-modulation distortion are minimal.

(5) Short-term level changes are preserved, so cues for sound localization based on interaural level differences are not markedly disrupted [45].

The disadvantages of slow-acting AGC are:

(1) Loudness perception is not restored to "normal" for all types of signals. For example, as mentioned earlier, loudness recruitment has the effect of magnifying perceived fluctuations in loudness of amplitudemodulated sounds [15]. Slow-acting AGC does not reduce the amplitude-modulation depth of sounds ex- cept for very low modulation rates [46], so this aspect of loudness perception is not restored to normal. In some slow-acting AGC systems, the output level changes only slightly with input level, and this may make it difficult for the user to judge the strength of sound sources, for example to judge whether a piece of music is being played piano or mezzo-piano.

(2) It may not deal effectively with situations where two voices or instruments alternate with markedly different levels.

(3) When there is a sudden drop in sound level, for example, when a forte passage is abruptly followed by a piano passage, the gain takes some time to increase. Hence the aid may appear to become "dead" for a while, and part of the piano passage is not heard.

(4) When trying to listen to one (target) voice or instrument in the presence of background voices/instruments, a normally hearing person can extract information about the target during the temporal dips in the background [47]. This process is called "listening in the dips." The information in the dips may be at a relatively low level. Slow-acting AGC is of limited benefit in this situation because the gain does not increase significantly during brief dips in the input signal.

The advantages of fast-acting AGC are:

(1) It can make loudness perception somewhat closer to "normal" if the input-output function is chosen appropriately. However, normal loudness is not quite achieved. When a person has loudness recruitment, an amplitude-modulated sound appears to fluctuate more than normal for modulation rates up to at least $32 \mathrm{~Hz}$ [15]. Even at the short end of the range of time constants used in hearing aids, fast-acting AGC does not reduce the depth of amplitude modulation for rates above about $10 \mathrm{~Hz}$ [46, 48-50]. Thus, dynamic aspects of loudness perception are not fully restored to normal.

(2) If many channels are used, fast-acting AGC can compensate for frequency-dependent changes in the degree of loudness recruitment more effectively than slowacting AGC. While slow-acting AGC can apply gain that is appropriate for the average level of the signal in each frequency channel, fast-acting AGC can also compensate for the short-term changes in signal level.

(3) Fast-acting AGC can restore the audibility of weak sounds rapidly following intense sounds. This provides the potential for listening in the dips.

(4) When two musical instruments or voices alternate with markedly different levels, fast compression can improve the audibility of the softer sound [51].

The disadvantages of fast-acting AGC are:

(1) It can introduce spurious changes in the shape of the temporal envelope of sounds (e.g., overshoot and undershoot effects) [52], although such effects can be 
reduced by delaying the audio signal by a small amount relative to the gain-control signal $[53,54]$.

(2) It can introduce spurious changes in amplitude of sounds gliding in frequency, such as formants in spoken or sung speech, as those sounds traverse the boundary between two channels. This happens mainly for systems in which the compression channels are formed using sharp, non-overlapping filters. The effect does not occur for systems in which the filters used to form the compression channels overlap and have rounded tops and sloping edges [55].

(3) It reduces intensity contrasts and the modulation depth of signals, which may have an adverse effect on the ability to hear out one musical instrument or voice from other instruments or voices [44].

(4) In a hearing aid with fast-acting AGC in many channels, the spectrum is flattened. This compounds difficulties produced by the reduced frequency selectivity that is associated with OHC damage [2].

(5) When the input signal to the AGC system is a mixture of different voices or instruments, fast-acting compression introduces "cross-modulation" between the voices/instruments because the time-varying gain of the compressor is applied to the mixture $[49,52,56]$. This may decrease the ability to perceptually segregate the voices/instruments. However, this effect appears to be small for musical sounds [44].

(6) When moderate levels of background sound are present (e.g., noise from ventilation and airconditioning systems), fast compression makes such sounds audible, and this can be annoying [57]. When the number of channels is small, steady background noises may appear to be modulated by "foreground" sounds such as music. This can also be annoying. However, this effect is reduced when the number of channels is increased.

(7) Cues for sound localization based on interaural level differences may be disrupted by the independent action of the AGC at the two ears [45, 58]. This effect can be avoided by synchronization of the AGC action across the two ears [58].

(8) When the AGC is very fast-acting, it can introduce harmonic and intermodulation distortion [59]. However, the AGC can be designed to minimize the perceptual effects of such distortion [54], and in practice harmonic and intermodulation distortion are not usually a significant problem in commercial hearing aids [50], except perhaps for very high input and output levels $[60,61]$.

On average, hearing-impaired people slightly prefer slow-acting AGC over fast-acting AGC for listening to music [62]. However, there are marked individual differences, and the reasons for these are not understood. Finally, AGC systems in hearing aids often operate in a manner that is far from optimal. In a recent survey, only $28 \%$ of hearing-aid users reported that they could hear soft passages in music without the louder parts being too loud [61].

\subsection{Multiple or Adaptive Time Constants}

Some hearing aids incorporate AGC with multiple time constants $[63,64]$ or time constants that adapt depending on the characteristics of the signal [65]. Such systems are generally designed so that they are slow-acting most of the time. However, if an intense sound suddenly occurs, the gain is rapidly reduced, to prevent loudness discomfort. If the intense sound lasts for only a short time, then the gain returns to the value that was operational before the intense sound occurred.

\section{COMPENSATION FOR REDUCED FREQUENCY SELECTIVITY AND IHC/NEURAL DYSFUNCTION}

Signal processing to compensate for the effects of reduced frequency selectivity by enhancing spectral contrast [66] or by enhancing spectral changes over time [67] has provided only limited benefits and has not been implemented in commercial hearing aids. The effects of dysfunction of IHCs/synapses/neurons cannot be compensated directly. If the loss of IHCs is severe but neural survival is good, then a cochlear implant may be more effective than a hearing aid. However, music perception via cochlear implants is generally rather poor [68].

The ability to "hear out" individual voices or instruments from a mixture could in principle be improved by use of directional microphone systems in hearing aids. However, the hearing aid does not "know" what voice or instrument the user wishes to attend to at any given moment, so generally an omnidirectional microphone is preferred for music listening.

\section{EFFECTS OF BANDWIDTH LIMITATIONS IN HEARING AIDS}

Most hearing aids do not provide significant gain for frequencies below about $200 \mathrm{~Hz}$ or above about $5000 \mathrm{~Hz}$ $[69,70]$. There have been several studies of the effects of bandwidth limitations on the sound quality of music as judged by hearing-impaired people. Ricketts et al. [71] obtained paired-comparison judgments of preference for (simulated) hearing-aid processed sounds using upper cutoff frequencies of 5.5 and $9 \mathrm{kHz}$. The sounds were a piece of music and a movie soundtrack. The gains were adjusted for each hearing-impaired listener using the NAL-NL1 fitting method [41]. Since this method does not give recommended gains for frequencies above $6 \mathrm{kHz}$, gains at high frequencies were based on a form of extrapolation. On average, the listeners showed a preference for the higher cutoff frequency, but not all listeners showed this. A steep slope of the audiogram (a rapid worsening of the absolute threshold with increasing frequency) was associated with a preference for the lower cutoff frequency.

It is not obvious why some hearing-impaired listeners preferred the lower cutoff frequency. One possibility is that these listeners were unused to hearing frequencies above about $6 \mathrm{kHz}$. When these high frequencies were amplified, 
the sound quality may have appeared unpleasant because of this unfamiliarity. If this were the case, such listeners might come to prefer a higher cutoff frequency after an acclimatization period [72]. Another possibility is that the fitting rule used for the high frequencies may have led to greater than normal loudness of the high frequencies for some listeners, leading to a "tinny" or harsh tone quality.

Moore et al. [62] examined the influence of upper cutoff frequency on preferences for music using a simulated five-channel compression hearing aid and the method of paired comparisons. The gains and compression ratios of the simulated hearing aid were set individually for each hearing-impaired listener, using the CAM2 method [73]. In one experiment, the upper cutoff frequency was set to 5, 7.5 or $10 \mathrm{kHz}$. There were substantial individual differences, some listeners consistently preferring the $7.5-$ and $10-\mathrm{kHz}$ cutoff frequencies and some consistently preferring the 5$\mathrm{kHz}$ cutoff frequency. As found by Ricketts et al. [71], a steep audiogram slope was associated with preference for the narrower bandwidth and a shallow slope was associated with a preference for the wider bandwidths.

The individual variability in preferences for cutoff frequency may have been related to the amount of highfrequency gain prescribed by CAM2; the gain might have been higher than preferred for some participants, leading them to prefer a lower cutoff frequency. To assess this possibility, preference judgments were obtained with the highfrequency gains of the simulated hearing-aid set both lower and higher than recommended by CAM2. For a classical music sample, the CAM2 gains and the reduced gains were approximately equally preferred, while the increased gains were not preferred. For a jazz sample, which had relatively less high-frequency energy, CAM2 gains tended to be preferred over either reduced or increased gains. However, the effects were small.

Overall, while normal-hearing listeners clearly prefer upper cutoff frequencies greater than $5 \mathrm{kHz}$ when listening to music [74], preferences among hearing-impaired listeners are less clear and vary markedly across listeners. Preferences for an upper cutoff frequency above $5 \mathrm{kHz}$ are associated with audiograms that do not have a steep slope [62, 71].

There have been relatively few studies of preferences for the lower cutoff frequency in hearing aids. However, Franks [75] showed that hearing-impaired listeners clearly preferred cutoff frequencies below $200 \mathrm{~Hz}$ when listening to music.

People with music-induced hearing loss often have normal or near-normal hearing at low frequencies. For such people, open-fit hearing aids are often used (the ear canal is not sealed), and low-frequency sounds are heard via leakage into the ear canal. In such cases, the low-frequency roll off of the hearing aid response is largely irrelevant. However, if a more closed fit is used, either because the user has a hearing loss at low frequencies or because a closed fit is required to reduce acoustic feedback at high frequencies, then the low-frequency response of the hearing aid becomes much more important. Tests using closed-fit hearing aids suggest that a lower cutoff frequency of about $50 \mathrm{~Hz}$ is re- quired for good sound quality when listening to music [76], consistent with the results obtained by Moore and Tan [74] for normal-hearing listeners.

\section{EFFECTS OF IRREGULAR FREQUENCY RESPONSE}

The frequency responses of hearing aids measured using a microphone close to the eardrum often show distinct ripples. For closed-fit hearing aids, these can be caused by resonances in the acoustical delivery system, for example, the tubing leading from a behind-the-ear hearing aid to the earmold. It is possible to reduce these peaks, smoothing the overall frequency response, by suitable modifications to the tubing and/or by the use of acoustic resistors [77, 78]. For open-fit hearing aids, ripples in the frequency response can be caused by the interference of (delayed) amplified sound from the hearing aid with (undelayed) sound leaking into the ear canal [79]. These ripples can be reduced by adjusting the gain in individual frequency channels, provided that the aid has many such channels. However, the ripples are difficult to eliminate completely, and, for a hearing aid with multichannel compression, the pattern of the ripples may change with input sound level.

A single broad peak of $12-15 \mathrm{~dB}$ in the frequency response around $3 \mathrm{kHz}$ is desirable, since this mimics the normal response of the outer ear. However, additional peaks and dips are not desirable and can have adverse effects on sound quality. To study the effects of frequency response irregularities in a well controlled manner, van Buuren et al. [80] artificially imposed peaks in the frequency response of a sound reproduction system via digital filtering, prior to delivery via headphones. The peaks were centered at 1.3 , 2.8 or $5.5 \mathrm{kHz}$ and had heights of 10,20 or $30 \mathrm{~dB}$ (note that the peaks occurring for real hearing aids typically have heights of $10 \mathrm{~dB}$ or less). The peaks were presented either singly or all three together. A reference condition without any such peaks was included. Frequency-dependent amplification was applied to ensure that the signals fell within the dynamic range of each hearing-impaired listener. Several music signals were used including: (1) flute, piano, and voice; (2) trumpet and orchestra; (3) drums, synthesizer, and voice; (4) piano. Listeners were asked to rate each sound sample on a scale ranging from "very unpleasant" to "very pleasant." Pleasantness ratings decreased systematically with increasing peak height and also tended to decrease with increasing center frequency of the peak. Multiple peaks led to lower pleasantness than a single peak. Even the smallest peaks used $(10 \mathrm{~dB})$ led to noticeable reductions in pleasantness for some of the music signals. This is consistent with results obtained for normal-hearing listeners $[74,81]$.

It can be concluded that the quality of music as perceived by hearing-impaired people is reduced by frequencyresponse irregularities when the peak-to-valley ratio in the response reaches $10 \mathrm{~dB}$, which can occur for some hearing aids. In addition, there are at least three benefits of smoothing the frequency response other than effects on sound quality: (1) it can reduce acoustic feedback; (2) it can reduce 
the distortion (including temporal distortion produced by rapid phase changes) that often occurs at frequencies around peaks in the response; (3) it can allow a greater proportion of the spectrum of the sound to be above threshold before the uncomfortable loudness level is reached.

\section{EFFECTS OF FEEDBACK CANCELLERS}

Sometimes, the sound generated by a hearing aid leaks back to the hearing aid microphone and sets up a sustained oscillation called acoustic feedback. This can be annoying to the user and to other people, it limits the maximum gain that can be applied, and it introduces distortion. Most modern hearing aids employ adaptive systems to cancel or filter out acoustic feedback [82]. However, such systems are not always fully effective. For example, in a survey, a third of hearing-aid users reported hearing acoustic feedback when listening to music [61]. Feedback occurred more often for respondents with mixed conductive and sensorineural hearing loss than for respondents with sensorineural hearing loss. Feedback also occurred more often for respondents with custom-made earmolds than for respondents with soft domes; the latter are often used with open-fitting hearing aids.

A limitation of acoustic feedback cancellation systems is that they can attempt to cancel real musical tones when the tones are steady (e.g., the sound from an accordion), and they can produce "after tones" when a musical tone suddenly stops. Some manufacturers use a small frequency shift at medium and high frequencies to reduce acoustic feedback. When an open fitting is used, this can lead to unpleasant beats produced by the interaction of the amplified sound and sound leaking into the ear canal.

\section{SPECIAL MUSIC PROGRAMS AND OTHER HEARING AID FEATURES}

Many hearing aids allow a special program to be set up for listening to music. The program can be selected either via a control on the hearing aid or via a remote control. It is not always obvious from the manufacturers' descriptions how the music program differs from the "standard" program, but common features seem to be: less aggressive noise reduction or no noise reduction at all; the use of slow compression; slowing down the speed of adaptation of any acoustic feedback canceller; and reduced directionality or no directionality of the microphones. It may also be possible to set up an extended low-frequency response for listening to music.

The effectiveness of music-listening programs is unclear. In a recent survey [61], $40 \%$ of respondents reported having a music program in their hearing aids. Reported experiences in listening to music did not differ markedly for respondents with and without a music program.

There is also uncertainty about the effect and importance of other features in hearing aids. For example, hearing aids differ in the number of frequency channels used for amplitude compression but there are few studies examining the effect of the number of channels on music perception.
Croghan et al. [83] compared simulated 3-channel and 18channel hearing aids and showed that the number of channels did not affect preferences for classical music, while for rock music 3 channels were preferred over 18 channels. The author is not aware of any studies of the effect of number of channels on music listening using intermediate numbers of channels.

Many manufacturers have introduced hearing aids that incorporate some form of frequency lowering [84]. The rationale is to provide information about high-frequency components of the input by shifting those components towards lower frequencies, where audiometric thresholds are usually better. In one form of frequency lowering, frequency components below a certain cutoff frequency are unaltered, but components above the cutoff frequency are shifted downwards by an amount that increases with increasing frequency. This is called "frequency compression." One might expect that frequency compression would make some musical notes appear to be "out of tune" with others and might make single musical tones sound strange because the upper frequency components are no longer at their "correct" harmonic frequencies. However, the cutoff frequency in such hearing aids is usually chosen to be above $2000 \mathrm{~Hz}$, and people are relatively insensitive to mistuning between the lower harmonics and the very high harmonics [85]. Results from a recent study suggest that mild amounts of frequency compression with a high cutoff frequency do not adversely affect music perception, but stronger compression or lower cutoff frequencies have detrimental effects [86].

With many hearing aids it is possible to send signals directly to the hearing aid via a wired or wireless link [87]. For example, signals may be sent from a smartphone, personal listening device, TV, or radio. This can produce a "cleaner" signal than when using the hearing-aid microphone, since effects of room reverberation and background noise are reduced or eliminated. However, wireless systems often involve significant time delays. Hence, they may not be suitable for listening to the TV or radio via an open-fitting hearing aid, since the asynchrony between the undelayed sound leaking to the ear canal and the delayed sound heard via the hearing aid can have disturbing effects; see Sec. 9 for discussion of the effect of delays.

\section{PROBLEMS WITH LIMITED DYNAMIC RANGE}

Hearing aids differ in the dynamic range of their input stages. The dynamic range can be limited by microphone noise, the maximum level that the microphone can handle before clipping occurs, the number of bits used in the analog-to-digital converter (ADC), and the gain and equalization applied to the microphone signal prior to the ADC. The limited dynamic range can be problematic when listening to music, especially live music, because of its very wide dynamic range. It may be even more of a problem for performers who play instruments that produce high output levels, such as drums.

Chasin [60] has argued that distortion in hearing aids for high input signal levels occurs mainly because of the limited dynamic range that can be handled by current ADCs. 
Schmidt [88] suggested reducing this problem by using microphones that are less sensitive at low frequencies. Hockley et al. [89] suggested shifting the dynamic range used by the ADC upwards to include higher sound levels. They found that this led to an overall improvement in musician's ratings of sound quality. At least one hearing aid manufacturer has introduced a hearing aid with an extended input dynamic range, leading to less distortion and improved sound quality for very high input levels [90].

\section{PROBLEMS WITH TIME DELAY}

Digital hearing aids delay the audio signal by $1-10 \mathrm{~ms}$, depending on the type of signal processing that is employed. One side-effect of such delays has already been mentioned (Sec. 5); for open-fit hearing aids (when the ear canal is left partly open) the interaction of the delayed and non-delayed sound can lead to ripples in the frequency response (comb filtering). However, the time delay itself can also lead to disturbing effects. For example, when the hearing-aid user speaks (or sings), there will be an asynchrony between the bone-conducted sound of the user's voice and the sound heard through the aid. In addition, the relative timing of the motor gestures and the sound will be abnormal. Stone and Moore [91] found that the disturbing effects of delay on perception of the user's own voice became significant when the delay reached about $20 \mathrm{~ms}$. For the delays typically found in commercial hearing aids $(<10 \mathrm{~ms})$, there was no significant disturbing effect. Another study [92] reported a small but significant disturbing effect of a $10-\mathrm{ms}$ delay (the largest used), but the rated disturbance was low for all delays.

There are few studies of the effects of delay when listening to music. Using an open-fit hearing aid and a single piece of music (the first $35 \mathrm{~s}$ of "The Way You Look Tonight" recorded by Brian Ferry), Groth and Søndergaard [92] found that a delay of $10 \mathrm{~ms}$ led to a significant disturbing effect for normal-hearing listeners but not for hearingimpaired listeners. Lester and Boley [93] investigated the effect of delay in live-monitoring scenarios, with monitoring either via loudspeaker "wedges" or in-ear monitors (IEM). They found that sensitivity to delay varied markedly across instruments; sensitivity was greatest for the saxophone and least for keyboards. They concluded that delays greater than $6.5 \mathrm{~ms}$ for wedges and greater than $1 \mathrm{~ms}$ for IEM would likely produce slight artifacts for some instruments, while delays greater than $16 \mathrm{~ms}$ for wedges and greater than $6.5 \mathrm{~ms}$ for IEM would probably produce a perception of actual delay for some instruments.

Some hearing aids produce a delay that is frequency dependent; usually the low frequencies are delayed more than the high frequencies [94]. This can make transient sounds appear to be smeared in time. For example a click may sound like a rapid frequency chirp. Stone and Moore [95] found that across-frequency delays of 9-15 ms led to significant disturbing effects on perception of the listener's own voice and when listening to speech. Also, delays of 15 $\mathrm{ms}$ or more had a significant deleterious effect on the ability to identify nonsense syllables. Kates and Arehart [94] measured the smallest detectable across-frequency delay for various types of signals, using both normal-hearing and hearing-impaired subjects. The lowest threshold of about 2 ms was found for the normal-hearing subjects and a click stimulus. Hearing-impaired subjects had a higher threshold for the click of about $5 \mathrm{~ms}$. The thresholds for speech signals were higher. The across-frequency delays in current hearing aids are usually less than the smallest detectable delay for speech stimuli [94].

\section{CONCLUSIONS}

Sound-induced hearing loss has effects beyond the obvious reduction of sensitivity to frequency components in the range 3 to $6 \mathrm{kHz}$. These effects include reduced frequency selectivity, loudness recruitment, and noisy transmission of signals from the ear to the brain, and they reduce the ability to discriminate and appreciate music.

Hearing aids compensate to some extent for threshold elevation and loudness recruitment, but they do not compensate for the effects of reduced frequency selectivity or noisy transmission of information from the ear to the brain. Furthermore, hearing aids can reduce sound quality because of several factors, including: limited frequency range; irregular frequency response; artifacts produced by feedback cancellation systems; frequency lowering (if activated); time delays, including frequency-dependent delays; and distortion for high input levels. The severity of these factors varies across hearing aids and may depend on how a given hearing aid has been fitted and adjusted.

\section{ACKNOWLEDGMENTS}

I thank Brian Glasberg, Tom Baer, Michael Stone, Aleksander Sek, Christian Füllgrabe, and Sara Madsen for their collaboration in the research reported here. I thank Tom Baer and three reviewers for helpful comments on an earlier version of this paper. This work was supported by the Medical Research Council (UK, grant number G0701870), The Engineering and Physical Sciences Research Council (UK, grant number RG78536), Action on Hearing Loss, Phonak, and Starkey.

\section{REFERENCES}

[1] M. C. Liberman and N. Y. S. Kiang, "Acoustic Trauma in Cats: Cochlear Pathology and Auditory-Nerve Activity," Acta Otolaryngol., vol. Suppl. 358, pp. 1-63 (1978).

[2] B. C. J. Moore, Cochlear Hearing Loss: Physiological, Psychological and Technical Issues, 2nd Ed. (Wiley, Chichester, 2007). http://dx.doi.org/ 10.1002/9780470987889

[3] S. G. Kujawa and M. C. Liberman, "Adding Insult to Injury: Cochlear Nerve Degeneration after 'Temporary' Noise-Induced Hearing Loss," J. Neurosci., vol. 29, pp. 14077-14085 (2009). http://dx.doi.org/10.1523/ JNEUROSCI.2845-09.2009 
[4] H. F. Schuknecht, Pathology of the Ear, 2nd Ed. (Lea and Febiger, Philadelphia, 1993).

[5] E. Borg, B. Canlon and B. Engström, "NoiseInduced Hearing Loss-Literature Review and Experiments in Rabbits. Morphological and Electrophysiological Features, Exposure Parameters and Temporal Factors, Variability and Interactions," Scand. Audiol., vol. 24, Suppl. 40, pp. 1-147 (1995).

[6] L. Robles and M. A. Ruggero, "Mechanics of the Mammalian Cochlea," Physiol. Rev., vol. 81, pp. 13051352 (2001).

[7] B. C. J. Moore, An Introduction to the Psychology of Hearing, 6th Ed. (Brill, Leiden, The Netherlands, 2012).

[8] B. R. Glasberg and B. C. J. Moore, "Derivation of Auditory Filter Shapes from Notched-Noise Data," Hear. Res., vol. 47, pp. 103-138 (1990). http://dx.doi.org/ 10.1016/0378-5955(90)90170-T

[9] B. C. J. Moore and B. R. Glasberg, "The Effect of Hearing Loss on the Resolution of Partials and Fundamental Frequency Discrimination," $J$. Acoust. Soc. Am., vol. 130, pp. 2891-2901 (2011). http://dx.doi.org/10.1121/1.3640852

[10] T. Baer and B. C. J. Moore, "Effects of Spectral Smearing on the Intelligibility of Sentences in the Presence of Interfering Speech," J. Acoust. Soc. Am., vol. 95, pp. 2277-2280 (1994). http://dx.doi.org/10.1121/1.408640

[11] E. P. Fowler, "A Method for the Early Detection of Otosclerosis," Arch. Otolaryngol., vol. 24, pp. 731-741 (1936). http://dx.doi.org/10.1001/archotol.1936. 00640050746005

[12] B. C. J. Moore, "Testing the Concept of Softness Imperception: Loudness Near Threshold for HearingImpaired Ears," J. Acoust. Soc. Am., vol. 115, pp. 31033111 (2004). http://dx.doi.org/10.1121/1.1738839

[13] E. Villchur, "Simulation of the Effect of Recruitment on Loudness Relationships in Speech," J. Acoust. Soc. Am., vol. 56, pp. 1601-1611 (1974). http://dx.doi.org/ $10.1121 / 1.1903484$

[14] B. C. J. Moore and B. R. Glasberg, "Simulation of the Effects of Loudness Recruitment and Threshold Elevation on the Intelligibility of Speech in Quiet and in a Background of Speech," J. Acoust. Soc. Am., vol. 94, pp. 2050-2062 (1993). http://dx.doi.org/10.1121/1.407478

[15] B. C. J. Moore, M. Wojtczak and D. A. Vickers, "Effect of Loudness Recruitment on the Perception of Amplitude Modulation," J. Acoust. Soc. Am., vol. 100, pp. 481-489 (1996). http://dx.doi.org/10.1121/1.415861

[16] B. C. J. Moore, "A Compact Disc Containing Simulations of Hearing Impairment," Br. J. Audiol., vol. 31, pp. 353-357 (1997). http://dx.doi.org/10.3109/ 03005364000000029

[17] J. Gaeth and T. Norris, "Diplacusis in Unilateral High Frequency Hearing Losses," J. Speech Hear. Res., vol. 8, pp. 63-75 (1965). http://dx.doi.org/10.1044/jshr.0801. 63

[18] E. M. Burns and C. Turner, "Pure-Tone Pitch Anomalies. II. Pitch-Intensity Effects and Diplacusis in Impaired Ears," J. Acoust. Soc. Am., vol. 79, pp. 1530-1540 (1986). http://dx.doi.org/10.1121/1.393679
[19] P. M. Sellick, R. Paluzzi and B. M. Johnstone, "Measurement of Basilar Membrane Motion in the Guinea Pig Using the Mössbauer Technique," J. Acoust. Soc. Am., vol. 72, pp. 131-141 (1982). http://dx.doi.org/ 10.1121/1.387996

[20] M. A. Ruggero, N. C. Rich, L. Robles and A. Recio, "The Effects of Acoustic Trauma, Other Cochlea Injury and Death on Basilar Membrane Responses to Sound," in A. Axelsson, et al. (ed.), Scientific Basis of Noise-Induced Hearing Loss (Thieme, Stockholm, 1996), pp. 23-35.

[21] K. S. Henry and M. G. Heinz, "Effects of Sensorineural Hearing Loss on Temporal Coding of Narrowband and Broadband Signals in the Auditory Periphery," Hear. Res., vol. 303, pp. 39-47 (2013). http://dx.doi. org/10.1016/j.heares.2013.01.014

[22] B. C. J. Moore, Auditory Processing of Temporal Fine Structure: Effects of Age and Hearing Loss (World Scientific, Singapore, 2014). http://dx.doi.org/10.1142/ 9064

[23] B. C. J. Moore, M. Huss, D. A. Vickers, B. R. Glasberg and J. I. Alcántara, "A Test for the Diagnosis of Dead Regions in the Cochlea," Br. J. Audiol., vol. 34, pp. 205-224 (2000). http://dx.doi.org/10.3109/03005364000000131

[24] B. C. J. Moore, "Dead Regions in the Cochlea: Conceptual Foundations, Diagnosis and Clinical Applications," Ear Hear, vol. 25, pp. 98-116 (2004). http://dx.doi. org/10.1097/01.AUD.0000120359.49711.D7

[25] K. Kähäri, G. Zachau, M. Eklöf, L. Sandsjö and C. Möller, "Assessment of Hearing Disorders in Rock/Jazz Musicians," Int. J. Audiol., vol. 42, pp. 279-288 (2003). http://dx.doi.org/10.3109/14992020309078347

[26] M. Huss and B. C. J. Moore, "Dead Regions and Noisiness of Pure Tones," Int. J. Audiol., vol. 44, pp. 599-611 (2005). http://dx.doi.org/10.1080/ 02640410500243962

[27] M. Huss and B. C. J. Moore, "Dead Regions and Pitch Perception," J. Acoust. Soc. Am., vol. 117, pp. 38413852 (2005). http://dx.doi.org/10.1121/1.1920167

[28] A. J. Noreña, "An Integrative Model of Tinnitus Based on a Central Gain Controlling Neural Sensitivity," Neurosci. Biobehav. Rev., vol. 35, pp. 1089-1109 (2010). http://dx.doi.org/10.1016/j.neubiorev.2010.11.003

[29] F. G. Zeng, "An Active Loudness Model Suggesting Tinnitus as Increased Central Noise and Hyperacusis as Increased Nonlinear Gain," Hear. Res., vol. 295, pp. 172179 (2012). http://dx.doi.org/10.1016/j.heares.2012.05.009

[30] R. W. Sweetow and J. H. Sabes, "Effects of Acoustical Stimuli Delivered through Hearing Aids on Tinnitus," J. Am. Acad. Audiol., vol. 21, pp. 461-473 (2010). http://dx.doi.org/10.3766/jaaa.21.7.5

[31] M. Pienkowski, R. S. Tyler, E. Rojas Roncancio, H. J. Jun, T. Brozoski, N. Dauman, C. B. Coelho, G. Andersson, A. J. Keiner, A. Cacace, N. Martin and B. C. J. Moore, "A Review of Hyperacusis and Future Directions: Part II. Measurement, Mechanisms, and Treatment," Am. J. Audiol., vol. 23, pp. 420-436 (2014). http://dx.doi.org/ 10.1044/2014_AJA-13-0037

[32] J. M. Kates, Digital Hearing Aids (Plural, San Diego, 2008). 
[33] ANSI, ANSI S3.22-2003, Specification of Hearing Aid Characteristics (American National Standards Institute, New York, 2003).

[34] E. Villchur, "Signal Processing to Improve Speech Intelligibility in Perceptive Deafness," J. Acoust. Soc. Am., vol. 53, pp. 1646-1657 (1973). http://dx.doi.org/ $10.1121 / 1.1913514$

[35] J. Verschuure, A. J. J. Maas, E. Stikvoort, R. M. de Jong, A. Goedegebure and W. A. Dreschler, "Compression and Its Effect on the Speech Signal," Ear Hear., vol. 17, pp. 162-175 (1996). http://dx.doi.org/10.1097/00003446199604000-00008

[36] P. E. Souza, "Effects of Compression on Speech Acoustics, Intelligibility, and Sound Quality," Trends Amplif., vol. 6, pp. 131-165 (2002). http://dx.doi.org/ $10.1177 / 108471380200600402$

[37] G. Keidser, H. Dillon, M. Flax, T. Ching and S. Brewer, "The NAL-NL2 Prescription Procedure," $\mathrm{Au}$ diol. Res., vol. 1:e24, pp. 88-90 (2011). http://dx.doi.org/ 10.4081/audiores.2011.e24

[38] B. C. J. Moore, B. R. Glasberg and M. A. Stone, "Development of a New Method for Deriving Initial Fittings for Hearing Aids with Multi-Channel Compression: CAMEQ2-HF," Int. J. Audiol., vol. 49, pp. 216-227 (2010). http://dx.doi.org/10.3109/14992020903296746

[39] R. M. Cox, G. C. Alexander, I. M. Taylor and G. A. Gray, "The Contour Test of Loudness Perception," Ear Hear., vol. 18, pp. 388-400 (1997). http://dx.doi.org/ 10.1097/00003446-199710000-00004

[40] B. C. J. Moore, "Use of a Loudness Model for Hearing Aid Fitting. IV. Fitting Hearing Aids with MultiChannel Compression so as to Restore "Normal" Loudness for Speech at Different Levels," Br. J. Audiol., vol. 34, pp. 165-177 (2000). http://dx.doi.org/10.3109/ 03005364000000126

[41] D. Byrne, H. Dillon, T. Ching, R. Katsch and G. Keidser, "NAL-NL1 Procedure for Fitting Nonlinear Hearing Aids: Characteristics and Comparisons with Other Procedures," J. Am. Acad. Audiol., vol. 12, pp. 37-51 (2001).

[42] S. D. Scollie, R. C. Seewald, L. Cornelisse, S. Moodie, M. Bagatto, D. Laurnagaray, S. Beaulac and J. Pumford, "The Desired Sensation Level Multistage Input/Output Algorithm," Trends Amplif., vol. 9, pp. 159-197 (2005). http://dx.doi.org/10.1177/108471380500900403

[43] H. G. Mueller, T. A. Ricketts and R. Bentler, Modern Hearing Aids: Pre-Fitting Testing and Selection Considerations (Plural, San Diego, CA, 2013).

[44] S. M. K. Madsen, M. A. Stone, M. F. McKinney, K. Fitz and B. C. J. Moore, "Effects of Wide DynamicRange Compression on the Perceived Clarity of Individual Musical Instruments," J. Acoust. Soc. Am., vol. 137, pp. 1867-1876 (2015). http://dx.doi.org/10.1121/1.4914988

[45] T. Van den Bogaert, T. J. Klasen, M. Moonen, L. Van Deun and J. Wouters, "Horizontal Localization with Bilateral Hearing Aids: Without Is Better Than With," J. Acoust. Soc. Am., vol. 119, pp. 515-526 (2006). http://dx.doi.org/10.1121/1.2139653

[46] M. A. Stone and B. C. J. Moore, "Syllabic Compression: Effective Compression Ratios for Signals Modulated at Different Rates," Br. J. Audiol., vol. 26, pp. 351-361 (1992). http://dx.doi.org/10.3109/03005369209076659

[47] A. J. Duquesnoy, "Effect of a Single Interfering Noise or Speech Source on the Binaural Sentence Intelligibility of Aged Persons," J. Acoust. Soc. Am., vol. 74, pp. 739-743 (1983). http://dx.doi.org/10.1121/1.389859

[48] M. A. Stone and B. C. J. Moore, "Effect of the Speed of a Single-Channel Dynamic Range Compressor on Intelligibility in a Competing Speech Task," J. Acoust. Soc. Am., vol. 114, pp. 1023-1034 (2003). http://dx.doi.org/10.1121/1.1592160

[49] M. A. Stone and B. C. J. Moore, "Side Effects of Fast-Acting Dynamic Range Compression that Affect Intelligibility in a Competing Speech Task," $J$. Acoust. Soc. Am., vol. 116, pp. 2311-2323 (2004). http://dx.doi.org/10.1121/1.1784447

[50] B. C. J. Moore, M. A. Stone and J. I. Alcántara, "Comparison of the Electroacoustic Characteristics of Five Hearing Aids," Br. J. Audiol., vol. 35, pp. 307-325 (2001).

[51] S. M. K. Madsen and B. C. J. Moore, "Effects of Compression and Temporal Asynchronies on the Detection of One Tone in the Presence of Another," J. Acoust. Soc. Am., vol. 135, pp. 2902-2912 (2014). http://dx.doi.org/10.1121/1.4870485

[52] M. A. Stone and B. C. J. Moore, "Quantifying the Effects of Fast-Acting Compression on the Envelope of Speech," J. Acoust. Soc. Am., vol. 121, pp. 1654-1664 (2007). http://dx.doi.org/10.1121/1.2434754

[53] C. E. Robinson and D. A. Huntington, "The Intelligibility of Speech Processed by Delayed Long-Term Averaged Compression Amplification," J. Acoust. Soc. Am., vol. 54, pp. 314 (1973). http://dx.doi.org/10.1121/1.1978243

[54] M. A. Stone, B. C. J. Moore, J. I. Alcántara and B. R. Glasberg, "Comparison of Different Forms of Compression Using Wearable Digital Hearing Aids," J. Acoust. Soc. Am., vol. 106, pp. 3603-3619 (1999). http://dx.doi.org/10.1121/1.428213

[55] E. Lindemann and T. L. Worrall, Continuous Frequency Dynamic Range Audio Compressor, USA Patent 6097824 (2000).

[56] M. A. Stone and B. C. J. Moore, "Effects of SpectroTemporal Modulation Changes Produced by Multi-Channel Compression on Intelligibility in a Competing-Speech Task," J. Acoust. Soc. Am., vol. 123, pp. 1063-1076 (2008). http://dx.doi.org/10.1121/1.2821969

[57] R. F. Laurence, B. C. J. Moore and B. R. Glasberg, "A Comparison of Behind-the-Ear High-Fidelity Linear Aids and Two-Channel Compression Hearing Aids in the Laboratory and in Everyday Life," $\mathrm{Br}$. J. Audiol., vol. 17, pp. 31-48 (1983). http://dx.doi.org/10.3109/ 03005368309081480

[58] I. M. Wiggins and B. U. Seeber, "Linking Dynamic-Range Compression across the Ears Can Improve Speech Intelligibility in Spatially Separated Noise," J. Acoust. Soc. Am., vol. 133, pp. 1004-1016 (2013). http://dx.doi.org/10.1121/1.4773862

[59] C. T. Tan and B. C. J. Moore, "Comparison of Two Forms of Fast-Acting Compression Using Physical 
and Subjective Measures," in Proceedings of the 18th International Congress on Acoustics (Kyoto, 2004).

[60] M. Chasin and N. S. Hockley, "Some Characteristics of Amplified Music through Hearing Aids," Hear. Res., vol. 308, pp. 2-12 (2014). http://dx.doi.org/10.1016/ j.heares.2013.07.003

[61] S. M. K. Madsen and B. C. J. Moore, "Music and Hearing Aids," Trends in Hearing, vol. 18, pp. 1-29 (2014). http://dx.doi.org/10.1177/2331216514558271

[62] B. C. J. Moore, C. Füllgrabe and M. A. Stone, "Determination of Preferred Parameters for MultiChannel Compression Using Individually Fitted Simulated Hearing Aids and Paired Comparisons," Ear Hear, vol. 32, pp. 556-568 (2011). http://dx.doi.org/ 10.1097/AUD.0b013e31820b5f4c

[63] B. C. J. Moore, B. R. Glasberg and M. A. Stone, "Optimization of a Slow-Acting Automatic Gain Control System for Use in Hearing Aids," Br. J. Audiol., vol. 25, pp. 171-182 (1991). http://dx.doi.org/10.3109/ 03005369109079851

[64] P. Nordqvist and A. Leijon, "Hearing-Aid Automatic Gain Control Adapting to Two Sound Sources in the Environment, Using Three Time Constants," J. Acoust. Soc. Am., vol. 116, pp. 3152-3155 (2004). http://dx.doi. org/10.1121/1.1793207

[65] P. J. Blamey, "Adaptive Dynamic Range Optimization (ADRO): A Digital Amplification Strategy for Hearing Aids and Cochlear Implants," Trends Amplif., vol. 9, pp. 77-98 (2005). http://dx.doi.org/10.1177/ 108471380500900203

[66] A. M. Simpson, B. C. J. Moore and B. R. Glasberg, "Spectral Enhancement to Improve the Intelligibility of Speech in Noise for Hearing-Impaired Listeners," Acta Otolaryngol., vol. Suppl. 469, pp. 101-107 (1990).

[67] J. Chen, T. Baer and B. C. J. Moore, "Effect of Spectral Change Enhancement for the Hearing Impaired Using Parameter Values Selected with a Genetic Algorithm," J. Acoust. Soc. Am., vol. 133, pp. 2910-2920 (2013). http://dx.doi.org/10.1121/1.4799807

[68] H. J. McDermott, "Music Perception with Cochlear Implants: A Review," Trends Amplif., vol. 8, pp. 49-82 (2004). http://dx.doi.org/10.1177/108471380400800203

[69] H. Aazh, B. C. J. Moore and D. Prasher, "The Accuracy of Matching Target Insertion Gains with Open-Fit Hearing Aids," Am. J. Audiol., vol. 21, pp. 175-180 (2012). http://dx.doi.org/10.1044/1059-0889(2012/11-0008)

[70] H. Dillon, Hearing Aids, 2nd Ed. (Boomerang Press, Turramurra, Australia, 2012).

[71] T. A. Ricketts, A. B. Dittberner and E. E. Johnson, "High Frequency Amplification and Sound Quality in Listeners with Normal through Moderate Hearing Loss," J. Speech Lang. Hear. Res., vol. 51, pp. 160-172 (2008). http://dx.doi.org/10.1044/1092-4388(2008/012)

[72] S. Gatehouse, "The Time Course and Magnitude of Perceptual Acclimatization to Frequency Responses: Evidence from Monaural Fitting of Hearing Aids," J. Acoust. Soc. Am., vol. 92, pp. 1258-1268 (1992). http://dx.doi.org/10.1121/1.403921
[73] B. C. J. Moore and C. Füllgrabe, "Evaluation of the CAMEQ2-HF Method for Fitting Hearing Aids with MultiChannel Amplitude Compression," Ear Hear, vol. 31, pp. 657-666 (2010).

[74] B. C. J. Moore and C. T. Tan, "Perceived Naturalness of Spectrally Distorted Speech and Music," J. Acoust. Soc. Am., vol. 114, pp. $408-419$ (2003). http://dx.doi. org/10.1121/1.1577552

[75] J. R. Franks, "Judgments of Hearing Aid Processed Music," Ear Hear., vol. 3, pp. 18-23 (1982). http://dx. doi.org/10.1097/00003446-198201000-00004

[76] C. Füllgrabe, B. C. J. Moore, D. J. van Tasell and M. A. Stone, "Effects of Bandwidth on Sound-Quality Preferences for Hearing Aids," Bull. Am. Aud. Soc., vol. 32, pp. 45 (2007).

[77] E. R. Libby, "Achieving a Transparent, Smooth, Wideband Hearing Aid Response," Hear. Inst., vol. 32, pp. 9-12 (1981).

[78] M. C. Killion, "Transducers, Earmolds and Sound Quality Considerations," in G. A. Studebaker and F. H. Bess (ed.), The Vanderbilt Hearing-Aid Report (Monographs in Contemporary Audiology, Upper Darby, PA, 1982), pp. 104-111.

[79] M. A. Stone, B. C. J. Moore, K. Meisenbacher and R. P. Derleth, "Tolerable Hearing-Aid Delays. V. Estimation of Limits for Open Canal Fittings," Ear Hear., vol. 29, pp. 601-617 (2008). http://dx.doi.org/ 10.1097/AUD.0b013e3181734ef2

[80] R. A. van Buuren, J. Festen and T. Houtgast, "Peaks in the Frequency Response of Hearing Aids: Evaluation of the Effects on Speech Intelligibility and Sound Quality," J. Speech Hear. Res., vol. 39, pp. 239-250 (1996). http://dx.doi.org/10.1044/jshr.3902. 239

[81] F. E. Toole and S. E. Olive, "The Modification of Timbre by Resonances: Perception and Measurement," $J$. Audio Eng. Soc., vol. 36, pp. 122-142 (1988 Mar.).

[82] D. J. Freed and S. D. Soli, “An Objective Procedure for Evaluation of Adaptive Antifeedback Algorithms in Hearing Aids," Ear Hear, vol. 27, pp. 382-398 (2006). http://dx.doi.org/10.1097/01.aud.0000224173. 25770.ac

[83] N. B. Croghan, K. H. Arehart and J. M. Kates, "Music Preferences with Hearing Aids: Effects of Signal Properties, Compression Settings, and Listener Characteristics," Ear Hear, vol. 35, pp. e170-184 (2014). http://dx.doi.org/10.1097/AUD.0000000000000056

[84] J. M. Alexander, "Individual Variability in Recognition of Frequency-Lowered Speech," Sem. Hear, vol. 34, pp. 86-109 (2013). http://dx.doi.org/10.1055/s-00331341346

[85] C. J. Darwin and R. P. Carlyon, "Auditory Grouping," in B. C. J. Moore (ed.), Hearing (Academic Press, San Diego, 1995), pp. 387-424. http://dx.doi.org/10.1016/ B978-012505626-7/50013-3

[86] B. S. Mussoi and R. A. Bentler, "Impact of Frequency Compression on Music Perception," Int. J. Audiol., vol. 54, pp. 627-633 (2015). http://dx.doi.org/ 10.3109/14992027.2015.1020972 
[87] A. Boothroyd, K. Fitz, J. Kindred, S. Kochkin, H. Levitt, B. C. J. Moore and J. Yanz. Hearing Aids and Wireless Technology 1-44-47 (2007).

[88] M. Schmidt, "Musicians and Hearing Aid Design-Is Your Hearing Instrument Being Overworked?" Trends Amplif., vol. 16, pp. 140-145 (2012). http://dx.doi.org/10.1177/1084713812471586

[89] N. S. Hockley, F. Bahaman and B. Fulton, "Analog-to-Digital Conversion to Accommodate the Dynamics of Live Music in Hearing Instruments," Trends Amplif., vol. 16, pp. 146-158 (2012). http://dx.doi.org/10.1177/1084713812471906

[90] F. Kuk, L. Chi-Chuen, P. Korhonen and B. Crose, "Evaluating Hearing Aid Processing at High and Very High Input Levels," Hear. Rev., vol. 21, pp. 32-35 (2014).

[91] M. A. Stone and B. C. J. Moore, "Tolerable Hearing-Aid Delays. II. Estimation of Limits Imposed During Speech Production," Ear Hear., vol. 23, pp. 325-338 (2002). http://dx.doi.org/10.1097/00003446200208000-00008

[92] J. Groth and M. B. Søndergaard, "Disturbance Caused by Varying Propagation Delays in Non-Occluding Hearing Aid Fittings," Int. J. Audiol., vol. 43, pp. 594-599 (2004).

[93] M. Lester and J. Boley, "The Effects of Latency on Live Sound Monitoring," presented at the 123rd Convention of the Audio Engineering Society (2007 Oct.), convention paper 7198 .

[94] J. M. Kates and K. H. Arehart, "Multichannel Dynamic-Range Compression Using Digital Frequency Warping," EURASIP J. Appl. Sig. Proc., vol. 18, pp. 3003 3014 (2005). http://dx.doi.org/10.1155/ASP.2005.3003

[95] M. A. Stone and B. C. J. Moore, "Tolerable Hearing-Aid Delays. III. Effects on Speech Production and Perception of Across-Frequency Variation in Delay," Ear Hear., vol. 24, pp. 175-183 (2003). http://dx.doi.org/10.1097/01.AUD.0000058106.68049.9C

\section{THE AUTHOR}

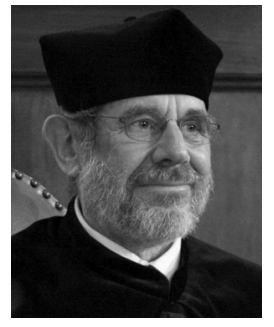

Brian C.J. Moore

Brian Moore is Emeritus Professor of Auditory Perception in the University of Cambridge. His research interests are: the perception of sound; mechanisms of normal hearing and hearing impairments; relationship of auditory abilities to speech perception; design of signal processing hearing aids for sensorineural hearing loss; methods for fitting hearing aids to the individual; design and specification of high-fidelity sound-reproducing equipment; perception of music and of musical instruments. He is a Fellow of the Royal Society of London, a Fellow of the Academy of Medical Sciences, a Fellow of the Acoustical Society of America, a Fellow of the Association for Psychological Science, and an Honorary Fellow of the Belgian Society of Audiology and the British Society of Hearing Aid Audiologists. He is a member of the Experimental Psychology Society (U.K.), the British Society of Audiology, The American Auditory Society, the Audio Engineering Society, and the Association for Research in Otolaryngology. He is President of the Association of Independent Hearing Healthcare Professionals (UK). He has written or edited 19 books and over 600 scientific papers and book chapters. He is an associate editor of the journal Hearing Research. He has been awarded the Littler Prize and the Littler Lecture of the British Society of Audiology, the Silver and Gold medals of the Acoustical Society of America, the first International Award in Hearing from the American Academy of Audiology, the Award of Merit from the Association for Research in Otolaryngology, and the Hugh Knowles Prize for Distinguished Achievement from Northwestern University. He is wine steward of Wolfson College, Cambridge. 